\title{
Cholesterol Embolization Syndrome after Primary Percutaneous Coronary Intervention
}

\author{
R.NHIRI*, Z.BAZID \\ Department of Cardiology, University Hospital of Mohammed VI, University Mohammed the First, Oujda, \\ Morocco
}

*Corresponding Author: R.NHIRI, Department of Cardiology, University Hospital of Mohammed VI, University Mohammed the First, Oujda, Morocco

\begin{abstract}
Background: Cholesterol embolization syndrome (CES) is a rare and under-diagnosed clinical entity. Vascular procedures such as angiography of vascular surgery have been identified as triggering factor. It frequently occurs in the elderly male population with extensive atherosclerosis. CES should be considered in the differential diagnosis of acute kidney injury (AKI) following angiography, as prognosis and treatment are completely different from contrast-induced nephropathy.
\end{abstract}

Case report: A 84-year-old man was admitted with new-onset bread and cyanosis in his feet. Toes and distal fingers were particularly affected. The patient had undergone coronary angiography and stent implantation to coronary arteries approximately 14 days prior, progressive deterioration in renal functions also developed gradually. Supportive therapy was provided in addition to hemodialysis sessions. But he died within 72 hours after massivegastrointestinal bleeding; probably the result of fresh cholesterol embolisation, and unfortunately cardiopulmonary resuscitation could not revive him.

Conclusion: In conclusion cholesterol embolization syndrome should be considered in any patient presenting with cutaneous features and renal failure after invasive vascular procedure.

\section{INTRODUCTION}

Cholesterol embolization syndrome (CES) is a is a multisystemic disease with immunological features, and a rare but an important cause of acute kidney injury (AKI) following angiography. CES is usually followed by an invasive vascular procedure, or anticoagulant or thrombolytic treatment. It may even occur spontaneously. One of the most important characteristic features of CES is AKI with poor prognosis causing high incidence of irreversible organ damage. $[1,2]$ Occasionally the presentation is atypical, with fever, myalgia, and multiorgan involvement mimicking systemic vasculitis.

Herein, a case of probable CES with cutaneous manifestation on all extremities renal failure, and multiorgan involvement, after performing primary percutaneous coronary intervention in patient with acute inferior myocardial infarction was presented.

\section{CASE Report}

A 84-year-old man was admitted with newonset bread and cyanosis in his feet. Toes and distal fingers were particularly affected. The patient had undergone coronary angiography and stent implantation to coronary arteries approximately 14 days prior, after which clopidogrel, acetylsalicylic acid, and statin treatments were begun. Smoking, 62 packyears, was present in personal medical history. Upon physical examination, his blood pressure was $110 / 60 \mathrm{mmHg}$ and heart rate was 132 beats/min, temperature was $36.4^{\circ} \mathrm{C}$, cyanotic lesions were present on the toes and distal parts of the fingers (Figure 1 and 2). All peripheral pulses were present on palpation. Lower extremity arterial Doppler USG was normal.

Electrocardiogram was in sinus rhythm and revealed sequelae of inferior necrosis. Echocardiography showed heterogeneous contractility with left ventricular ejection fraction of $35 \%$. Left ventricular thrombi were not detected.

In biochemical analysis, total leukocyte: 21250 $\mathrm{mm} 3$; eosinophil: 800/mm3 (reference range: 0 500/mm3); hemoglobin: $17.1 \mathrm{~g} / \mathrm{dL}$; platelets: 27000/mm3; with presence of platelet clumps at 
the smear; his initial serum creatinine level (before angioplasty) aroused from $12 \mathrm{mg} / \mathrm{L}$ to $29 \mathrm{mg} / \mathrm{L}$; hyperkaliemia to $7 \mathrm{mmol} / \mathrm{l}$; urea: 2.6 $\mathrm{g} / \mathrm{l}$; increased erythrocyte sedimentation rate (68 mm/h); CRP: $195 \mathrm{mg} / \mathrm{L}$.

Rheumatologic markers were negative. Serum complement levels $(\mathrm{C} 3, \mathrm{C} 4)$ were low.

Close chronological association with coronary intervention, the signs and the laboratory results suggested the diagnosis of probable CES. Antiinflammatory therapy or corticosteroid therapy was not started due to the acute myocardial infarction. Antiplatelet therapy was maintained. High dose statin therapy was continued. Supportive therapy was provided by effective hydration and wound care.

The renal function continues to deteriorate requiring hemodialysis, and the patient was presented an abdominal pain, diarrhea and gastrointestinal bleeding;

Unfortunately, the evolution was unfavorable, and he died within 72 hours after massivegastrointestinal bleeding, probably the result of fresh cholesterol embolisation.

\section{DISCUSSION}

Cholesterol embolization is a serious complication of atherosclerosis. Mainly, vascular manipulations during endovascular procedures or vascular surgery can release cholesterol crystals from atheromatous plaques. We hereby described a case of CES with renal and cutaneous involvement affecting extremities after performing coronary intervention in patient with acute myocardial infarction.

CES is a systemic disorder that can spread to multiple organs resulting renal failure, heart failure, gastrointestinal ischemia and skin necrosis. Most affected organs related to CES are the brain, the kidney, the gastrointestinal tract and the skin. Central nervous system involvement may lead to confusion or memory loss. [3]

In our patient, there was no symptom or sign of brain injury. The retinal artery occlusion may present with Hollenhorst plaques on retina examination. Embolization to the kidneys has usually patchy distribution. Elevation of serum creatinine and proteinuria are the main laboratory findings. [4] In the present case, serum creatinine level increased rapidly requiring the use of extrarenal depuration.
Cutaneous manifestations of CES include livedo reticularis, gangrene, cyanosis, nodules, macule, ulceration and purpura. Purple or blue discoloration of the foot lead to call " blue toe syndrome " synonymous with CES. Cutaneous manifestations almost always located to the lower extremities and rarely extended to upper extremities. [5] In our case bluish lesions were observed

not only on the feet but also on the hands. Risk factors for CES are male gender, smoking, advanced age, hypertension and aortic aneurysm. Development of clinical event is triggered by invasive procedures of aorta, anticoagulation and thrombolysis. [6] Mainly, a plaque located at proximal, large-calibre artery is ruptured by iatrogenic trauma such as catheter manipulation or spontaneously. Embolization of plaque debris to small or medium sized arteries leads to mechanical occlusion and foreign body inflammatory response which result in endorgan damage. Common source of atheroembolism is the abdominal aorta thus embolism to upper extremities is unusual. CES during coronary angioplasty is uncommon with the incidence of $0.6 \%$. [7]

In the diagnosis of CES, constitutional symptoms and signs are frequent in addition to specific end-organ damage presentation. Leukocytosis with hypereosinophilia, arise in erythrocyte sedimentation rate and C-reactive protein and a decrease in serum complement level may be seen in laboratory tests. Biopsy for any target organ such as kidney or skin could also be obtained for histopathological confirmation. However, none of the test is specific for CES so clinical suspicion is very crucial in the diagnosis of CES. Skin biopsy was not performed in this case. Although pathological findings may be seen in cases, cholesterol cleft identification is limited. Furthermore, in delayed biopsies necrotizing vasculitis may also be seen. [8]

In differential diagnosis, sepsis disseminated intravascular coagulation (DIC) and heparininduced thrombocytopenia (HIT) should also keep in mind. While the prolonged activated partial thromboplastin time and prothrombin time and also low levels of protein-C and antithrombin are associated with DIC, coagulation tests is usually normal in HIT with increase in platelet count after cessation of heparin. Positive blood culture and positive sepsis criteria are main determinants in sepsis without DIC. As CES is a clinical entity, in this case diagnosis of CES is confirmed by using 
symptoms, signs, laboratory findings and close chronological association with coronary intervention.

There is no widely acceptable treatment for CES. Supportive therapy is aimed at end-organ damage. Risk factor modification for atherosclerosis should always be considered such as smoking, hypertension, diabetes mellitus and hyperlipidemia. Corticosteroid use may control the inflammation. Statins are considered as key therapy for stabilising atherosclerotic plaque. Antiplatelet agents, reninangiotensin system blockers should be considered for therapy. Surgical repair of the diseased aorta should also be used for therapy.[9] In our patient, antiplatelet therapy maintained due to acute myocardial infarction and coronary stenting.

Atheroemboli to the gastrointestinal tract represent an important often unsuspected cause of abdominal manifestations. Involvement of the digestive system varies from $18.6-48 \%$ and can occur at any site along its length, producing many, often misleading, presentations (10$\underline{11}, \underline{12})$. Usually, the prognosis of patients with gastrointestinal disease is poor; the overall death rate is high. In our case the mode of presentation of cholesterol crystal embolism to the bowel was hemorrhage and abdominal pain. Gastrointestinal bleeding most often results from superficial mucosal ulcerations, erosions or mucosal infarcts.

\section{Conclusion}

It is vital to consider the diagnosis cholesterol embolism whenever an elderly patient with pronounced atherosclerosis develops progressive renal failure and multiorgan failure. Especially after invasive vascular procedure. There is no effective therapy. The key is prophylaxis, which means identifying patients at risk prior to invasive vascular procedures.

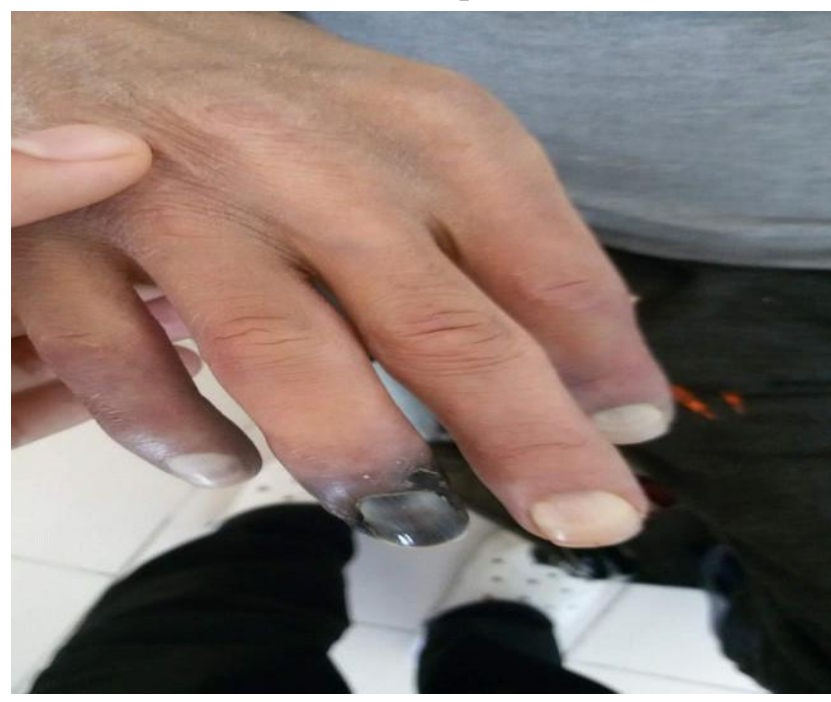

Figure1: Clinical image of purple coloured skin manifestation on the hand.

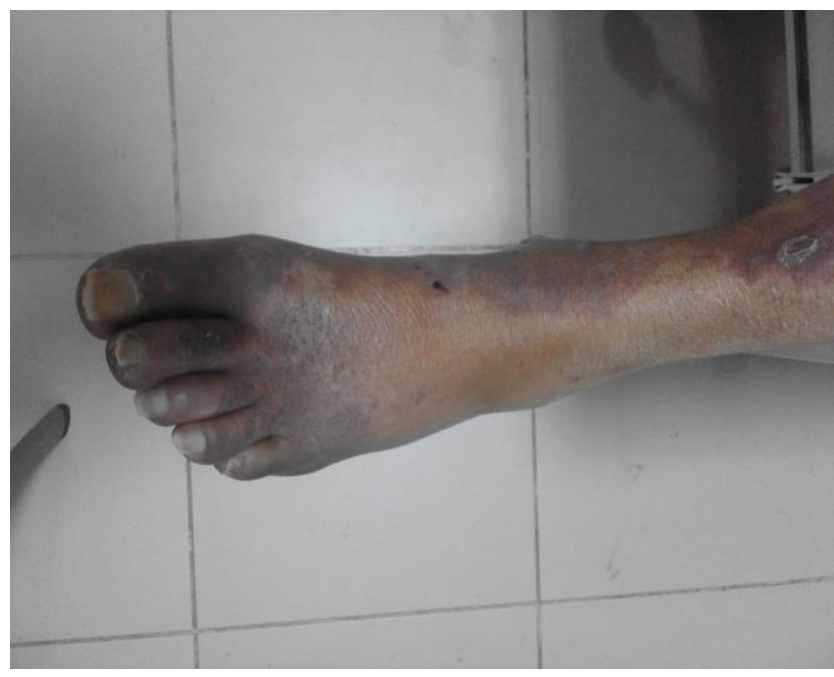

Figure2: Clinical image of cyanotic lesions on the feet. 


\section{REFERENCES}

[1] Scolari F, Ravani P. Atheroembolic renal disease. Lancet 2010; 375:1650-60.

[2] Kronzon I, Saric M. Cholesterol embolization syndrome. Circulation 2010; 122:631-41.

[3] Kronzon I, Saric M. Cholesterol embolization syndrome. Circulation 2010; 122:631-641.

[4] Scolari F, Tardanico R, Zani R, et al. Cholesterol crystal embolism: a recognizable cause of renal disease. Am J Kidney Dis 2000; 36:1089-1109.

[5] Falanga V, Fine MJ, Kapoor WN. The cutaneous manifestations of cholesterol crystal embolization. Arch Dermatol 1986;122:11941199

[6] Funabiki K, Masuoka H, Shimizu H, et al. Cholesterol crystal embolization (CCE) after cardiac catheterization: a case report and a review of 36 cases in the Japanese literature. Jpn Heart J 2003;44:767-774
[7] Johnson LW, Esente P, Giambartolomei A, et al. Peripheral vascular complications of coronary angioplasty by the femoral and brachial techniques. Catheter Cardiovasc Diagn 1994;31:165-172

[8] Grau R. Pseudovasculitis: mechanisms of vascular injury and clinical spectrum. CurrRheumatol Rep 2002;4:83-89

[9] Belenfant X, Meyrier A, Jacquot C. Supportive treatment improves survival in multivisceral cholesterol crystal embolism. Am J Kidney Dis 1999;33:840-850

[10] Kassirer J. Atheroembolic renal disease. N Engl J Med. 1969; 280:812-18. [PubMed]

[11] Lye WC, Cheah JS, Sinniah R. Renal cholesterol embolic disease. Case report and review of the literature. Am J Nephrol. 1993;13:489-93. [PubMed]

[12] Scolari F, Ravani P, Gaggi R, et al. Circulation. 2007. The challenge of diagnosing atheroembolic renal disease: clinical features and prognostic factors. (in press) [PubMed].

Citation: R.NHIRI, Z.BAZID, Cholesterol Embolization Syndrome after Primary Percutaneous Coronary Intervention. ARC Journal of Cardiology. 2021; 7(1): 01-04. Doi: doi.org/10.20431/2455-5991.0701001.

Copyright: (C) 2021 Authors. This is an open-access article distributed under the terms of the Creative Commons Attribution License, which permits unrestricted use, distribution, and reproduction in any medium, provided the original author and source are credited. 